\title{
BMJ Open Evaluation of the decision-making process underlying the initial off-label use of vaccines: a scoping review protocol
}

\author{
Dieynaba Diallo (10 , , ${ }^{1,2}$ Caroline Quach (1) ${ }^{1,2}$
}

To cite: Diallo D, Quach C. Evaluation of the decisionmaking process underlying the initial off-label use of vaccines: a scoping review protocol. BMJ Open 2021;11:e042748. doi:10.1136/ bmjopen-2020-042748

- Prepublication history and additional material for this paper is available online. To view these files, please visit the journal online (http://dx.doi.org/10. 1136/bmjopen-2020-042748).

Received 13 July 2020 Revised 31 October 2020 Accepted 09 February 2021

Check for updates

(c) Author(s) (or their employer(s)) 2021. Re-use permitted under CC BY-NC. No commercial re-use. See rights and permissions. Published by BMJ.

${ }^{1}$ Centre de Recherche, Centre Hospitalier Universitaire SainteJustine, Montreal, Quebec, Canada

${ }^{2}$ Microbiology, Infectious Diseases, and Immunology, University of Montreal Faculty of Medicine, Montreal, Quebec, Canada

Correspondence to

Dr Caroline Quach;

c.quach@umontreal.ca

\section{ABSTRACT}

Introduction Vaccination has become a central part of public health prevention. Vaccines are introduced after licensure by national regulatory authorities, whereas recommendations for use of licensed vaccines are made by national or international advisory committees and may include off-label use. The methodological and decisionmaking processes that are used to assess novel initial offlabel vaccine use are unclear. This review aims to examine the off-label assessment processes to map evidence and concepts used in the decision-making process and present a common approach between all recommendations and specifics of each decision.

Methods and analysis The methodological framework described at the Joanna Briggs Institute will be applied to this scoping review. A search strategy was developed, in collaboration with an experienced senior health research librarian, by combining Mesgarpour's highly sensitive search strategies. Peer-reviewed and grey literature will be systematically identified using PubMed, Medline and EMBASE; governmental agency and pharmaceutical websites; and search engines, such as Google Scholar. Reports and studies on off-label vaccine use in public health will be included. Screening will be independently undertaken by two reviewers. Data will be extracted using a standard form. Results will be narratively summarised to highlight relevant findings and guide the development of an analytical framework for off-label vaccination recommendations.

Ethics and dissemination This research does not require ethical approval. This scoping review will provide decision-making elements and a synthesis of knowledge on vaccines off-label use. Findings will be relevant to decision-makers/advisory committees and public health. These will be disseminated through peer-reviewed articles and conferences.

\section{INTRODUCTION}

\section{Background and rationale}

Infectious diseases are the most common cause of deaths worldwide, killing more than 17 million people a year, ${ }^{1}$ although many are preventable or curable diseases. In 2016, lower respiratory infections remained the deadliest communicable disease and were among the top 10 causes of deaths, with diarrhoea and
Strengths and limitations of this study

- Strengths of this review comprise the substantial significance of mapping the decision-making processes and methods used for off-label vaccine recommendations.

- The use of recognised scoping review methodology.

- A search strategy developed in collaboration with an experienced senior health research librarian.

- Systematic screening and extraction of data independently conducted in duplicate.

- Off-label vaccine use established practices, not published in an official form by national authorities, potentially represent a limitation for this review.

tuberculosis, and accounted for a total of 5.7 million deaths worldwide that year ${ }^{2}$ : in low-income countries, more than half of all deaths were caused by conditions involving communicable diseases (Crude death rate per 100000 population: lower respiratory infection 76; diarrhoeal diseases 58; HIV/ AIDS 44.5; Malaria 38; Tuberculosis 34.5). In Canada, infectious and parasitic diseases were responsible for $1.6 \%$ of all deaths in $2018 .^{3}$

In the course of time, numerous vaccines have been developed to prevent diseases. In $2018,85 \%$ of infants worldwide had received three doses of polio vaccine to protect them against poliomyelitis-a highly infectious viral disease that can cause irreversible paralysis. ${ }^{4}$ In the same year, an estimated $35 \%$ of infants globally were protected against rotaviruses, the most common cause of severe diarrhoeal disease among children worldwide. The global coverage of the third dose of the pneumococcal vaccine was estimated at $47 \%$ in 2018 . Thus, vaccination has become a central part of public health preventive measures against morbidity, disability and mortality.

The vaccine industry has become highly regulated through licensure. ${ }^{5}$ The national 
regulatory authorities (NRA) licence a vaccine after clinical trial data submitted by the manufacturer confirm the vaccine safety and efficacy for its intended use. Every vaccine has specific indications of use that are mentioned when introduced to the market. The vaccine's label provides information, such as the name, formulation, dosage, route of administration, age, indications and usage, and contraindications or other information unique to the vaccine. ${ }^{6}$

After vaccines are licensed, national immunisation programmes that are implemented by healthcare practitioners and clinicians may include these vaccines and will describe, for each vaccine, the NRA-approved prescribing information. ${ }^{5}$ Subsequently, expert technical advisory committees-national or international-will make recommendations based on several additional elements, such as disease epidemiology (eg, serotype distribution), vaccine effectiveness/efficacy, vaccine impact, cost, supply or programme optimisation. ${ }^{7}$ Very often, however, recommendations for the use of a licensed vaccine can be for off-label indications, ${ }^{8}$ which involves the use of a licensed vaccine on a dosage, schedule or within a population outside the indications approved by a regulatory body.

The unlabelled use of vaccines (unlicensed) is different from off-label use, which results from recommendations for licensed vaccines and is supported by critically appraised evidence. There are known off-label recommendations that are reported in the literature. For example, at licensure, Prevnar-7 (PnC7 conjugated 7-valent pneumococcal vaccine) was approved in a $3+1$ schedule. In Canada, the National Advisory Committee on Immunisation recommended an off-label schedule of $2+1$ instead of the approved $3+1 .{ }^{9}$ Another example is REPEVAX (diphtheria and tetanus toxoids, acellular pertussis adsorbed and inactivated poliovirus vaccine), which is not indicated for use during pregnancy because its effect on embryofetal development has not been assessed. REPEVAX has not been evaluated in fertility studies. ${ }^{10}$ However, no teratogenic effect of vaccines containing diphtheria or tetanus toxoids, or inactivated poliovirus have been observed following use in pregnant women, and there is some post-marketing information on the safety of administering REPEVAX to pregnant women. Therefore, its use for pregnant women in the UK is off-label, but considered the approved summary of product characteristics (SmPC) (The SmPC is used by healthcare professionals, such as doctors, nurses and pharmacists, and explains how to use and prescribe a medicine. SmPCs are written and updated by pharmaceutical companies and are based on their research and product knowledge). ${ }^{11}$

RotaTeq (Rotavirus Vaccine, Live, Oral, Pentavalent) was licensed in February $2006^{12}{ }^{13}$ by the US Food and Drug Administration (FDA) for the prevention of rotavirus gastroenteritis, caused by types G1, G2, G3 and G4, in infants in the age range of 6-32 weeks, administered as a 3-dose series. In the USA, the Advisory Committee on Immunisation Practices recommended routine oral vaccination of infants with 3 doses of this rotavirus vaccine at ages 2, 4 and 6 months. ${ }^{14}$ Rotarix (Rotavirus vaccine, live, attenuated) was licensed in February $2006^{1516}$ by the European Medicines Agency (EMA) for use in the European Union in babies 6-24 weeks of age to protect them against gastroenteritis (diarrhoea and vomiting) caused by rotavirus infection. Experts are investigating the possibility of waivers for patients younger than or older than 6 and 32 weeks of age, respectively, ${ }^{17}$ or for different dosing schedules of rotavirus vaccines. ${ }^{18}$

Thus, off-label use of vaccines exists and is feasible when supported by scientific evidence. Among diverse populations and given the large number of vaccines, many considerations and elements should be assessed before a recommendation is made. However, for novel off-label vaccine use, the evaluation process does not rely on previous off-label recommendations of one vaccine and requires new evidence to support a recommendation.

\section{Previous studies}

We searched the literature to verify whether studies had examined the process for evaluating the initial off-label use of a vaccine or its recommendation. A pilot selection of databases and relevant studies identified mainly randomised controlled trials (RCTs) and systematic reviews on individual vaccines. Systematic reviews were conducted to evaluate the impact ${ }^{1920}$ and effectiveness $^{21-23}$ of vaccines, mortality ${ }^{24}$ and morbidity. ${ }^{25}$ Moreover, we searched the literature for scoping reviews $(\mathrm{ScR})$ of off-label use of vaccine, to check whether similar work, as comprehensive as the research we intend to undertake, had been conducted. Several papers reported off-label recommendations that had been implemented by public health decision-makers, ${ }^{8918}$ but few have investigated the methodology behind the process for off-label recommendations. ${ }^{26}{ }^{27}$ To our knowledge, no ScR has thus far been conducted with a spectrum of data elements, synthesised for decision making, considered in a recommendation for the off-label use of vaccines in a public health programme. Further in-depth research is needed to map out approaches, evidence and recommendations for offlabel vaccine use. Key elements of national and global importance will be highlighted in this review. ${ }^{28} 29$

\section{Aims and objectives \\ Aim}

To synthesise the knowledge around off-label use of vaccines in an initial assessment process at a global level. The ScR method will allow us to examine peer-reviewed and grey literature and to map the broad topic of the offlabel use of vaccine in a rigorous, systematic and reproducible manner. A greater understanding of the nature of evidence that supports vaccine off-label use recommendations may lead to feasible and improved decision making in public health. This ScR is the first step of a three-phase research plan which includes a survey and a focus group in the second and third phase respectively towards the development of an analytical framework for off-label vaccine recommendations. 
We define the initial assessment as the process that occurs after a vaccine has been licensed and wherein an off-label recommendation from an expert committee is implemented in a public programme within a jurisdiction, before any other global off-label recommendation has been made for the same vaccine. To identify such processes, we will use the vaccine licensure date as a starting point and search for any published off-label recommendation that chronologically flows from it.

\section{Objectives}

1. To map the field of methods and concepts used in the decision-making process of a recommendation about off-label vaccination.

2. To identify and describe the different assessment processes that lead to a decision and its implementation of initial off-label vaccine use.

3. To identify and validate the recommendations on offlabel vaccination that have been reported by advisory committees and which may help plan immunisation programmes.

4. To identify and summarise the range of evidence that inform the development of recommendations across different off-label types and characteristics.

5. To present a common approach between all initial offlabel use of vaccine recommendations and the specific aspects of each decision.

6. To provide a clear definition of the off-label use of vaccines.

7. To highlight relevant findings that will guide the conceptualisation of an analytical framework for off-label vaccine use.

\section{Review question}

What are the evidences used by public health experts in recommending off-label use of vaccines in a vaccination programme?

\section{METHODS AND ANALYSIS \\ ScR design}

This study will follow the Joanna Briggs Institute (JBI) ${ }^{30}$ methodological approaches for a ScR, as described by Peters et al in Chapter 11 of the fourth Edition of the reviewer's manual. The JBI framework involves:

1. Defining and aligning the objective/s and question/s.

2. Developing and aligning the inclusion criteria with the objective/s and question/s.

3. Describing the planned approach to evidence searching, selection.

4. Searching for the evidence.

5. Selecting the evidence.

6. Extracting the evidence.

7. Charting the evidence.

8. Summarising the evidence in relation to the objective/s and question/s.

9. Consultation of information scientists, librarians and/ or experts (throughout).
Vaccines that will be included in the ambit of this $\mathrm{ScR}$ have been identified. This ScR has been initiated as the protocol was submitted for publication. Reporting will be conducted in accordance with the Preferred Reporting Items for Systematic Reviews and Meta-Analyses Extension for ScR (PRISMA-ScR) checklist. ${ }^{31}$

\section{Review registration}

At present, ScR protocols are ineligible for registration in the PROSPERO database.

This review title has been registered with Open Science Framework. ${ }^{32}$

\section{Patient and public involvement}

There will be no patient or public involvement in this review. However, patient/public involvement will be a part of the third phase of the research plan, during a focus-group interview to be conducted after the results of this review are reported.

\section{Inclusion criteria}

There are 26 vaccine-preventable diseases (VPD) that are part of a routine immunisation programme for which a vaccine is available, and these will be included in our review:

- Cholera.

- Dengue.

- Diphtheria.

- Hepatitis A.

- Hepatitis B.

- Hepatitis E.

- Hemophilus influenzae type b.

- Human papillomavirus.

- Influenza.

- Japanese encephalitis.

- Malaria.

- Measles.

- Meningococcal meningitis.

- Mumps.

- Pertussis.

- Pneumococcal invasive disease.

- Poliomyelitis.

- Rabies.

- Rotavirus.

- Rubella.

- Tetanus

- Tickborne encephalitis.

- Tuberculosis.

- Typhoid.

- Varicella.

- Yellow fever.

\section{Search strategy}

\section{Search terms and strategy}

A comprehensive and structured search of the literature will be conducted. For documents identification, two search strategies will be developed: one for the grey literature and the other for published studies. 
Table 1 Population, concept and context elements - Review inclusion criteria Inclusion

$\begin{array}{ll}\text { Types of } & \text { Public health immunisation is a broad endeavour, and it is aimed at } \\ \text { participants } & \text { the entire population. All strata and categories of individuals will be } \\ & \text { suitable for inclusion: males and females of any age group, condition or } \\ & \text { profession, as long as the off-label schedule is applicable to the group in } \\ & \text { a public health recommendation. }\end{array}$

Concept

Context

Types of sources
Methodically, any indication of use that would be different from the prescribing information provided in the label of a vaccine should be considered off-label immunisation. The most frequent off-label recommendations are for doses, population groups, indications, posology or injection site, ${ }^{78}$ but should not be limited to these aspects. An objective of our review is to identify all existing recommendations that address off-label vaccination in public health. The implementation of the recommendation for off-label vaccine use is considered an outcome when recommendations are part of published vaccination programmes. The review uses the vaccine licensure as a starting point to determine the eligibility of a paper, and the label is considered the baseline for each vaccine. Various terms and definitions may have been used through the years. However, as 'off-label' is a relatively new term that has been introduced in search engines in approximately 2010, the review intends to provide a clear definition for off-label vaccine use.

\section{Exclusion}

- Non-human subjects (eg, preclinical studies). Phase I, II or III clinical trials, unless it is used as an evidence in a recommendation

- Self-reporting of off-label-use of vaccine at the individual patient/ physician level, as this is not representative of a public health approach (no case report).

- Unlabelled vaccine use

- Superfast-track approval is not considered off-label use.

- Non-adherent behaviours that result in different dosing are not considered as off-label use.

Off-label recommendations will be broadly sought from within the global No exclusion criteria. context of immunisation. There will be no limitation in the geographic location or in the settings. This review is intended to map the evidence that emerges from any context, including pandemics and shortages, and to provide findings that support the development of an analytical framework applicable to any context.

\section{Any and all documents included in the decision process of the initial No exclusion criteria.}

off-label use of vaccine recommendations will be included in this review.

The reference lists of identified reports will be manually searched for additional studies.

All types of studies and documents: product monographs, official documents, recommendations (NITAG, SAGE, etc), health authority vaccine updates, and accessible documentary evidence submitted for licensing (from clinical trials: quality, safety, and efficacy data), or from studies made after licensing. Any valuable written sources will be included to supplement the information on the vaccines.

The period considered will be from the date of vaccine first licensing for the country, for each vaccine. Documents in all languages will be eligible at the initial phase. If texts are available in languages other than English or French, they will be translated and included in the review.

NITAG, National Immunisation Technical Advisory Group; SAGE, WHO Strategic Advisory Group of Experts.

For the grey literature, ${ }^{33}$ a search will be conducted for each vaccine's product monograph from pharmaceuticals, licensure, national vaccine updates or accessible documentary evidence submitted for licensing, identified by NRAs and organisations that proceeded to regulatory approval at the national or international level. Expert committees that make recommendations for off-label vaccines use will be identified.

A combination of terms-VPD, vaccine names and licensure-will be used to search official publications and all documents on the evaluation process, recommendations, fundamental decisive factors and programme implementation. All documents describing the decision-making process of off-label vaccine recommendation in a public programme, from the evaluation process by the expert committee to the decisive elements that enabled the health authority to implement the recommendation, or otherwise, into the vaccination programme. If necessary, we will contact the authors of the off-label decision for additional information.

The other search strategy will include a combination of two major concepts: off-label use (main concept) and vaccines (second concept). For the off-label concept, we will use Mesgarpour et al $\mathrm{s}^{34}{ }^{35}$ highly sensitive search strategy to retrieve as many documents as possible. The specificity of the search strategy will increase when 
combined with the second concept-vaccines and each VPD name. The outcome concept will not be included in the search strategy, as it could possibly restrict the number of papers. A medical librarian with experience in electronic database searches has worked with the research team and helped perfect the search strategy (online supplemental).

The exposure terms will be medical subject heading or EMBASE subject headings (EMTREE) that describe the off-label use, plus terms that describe vaccines, combined with the AND Boolean term. Word strings will be identified in the titles and abstracts of relevant documents. Variations of these words will be searched as free text.

\section{Databases and other sources to be searched}

The search will be conducted in the databases listed below for all published documents, without date or study type restrictions, by using the prespecified search terms.

For the grey literature, ${ }^{33}$ the sources to be searched are the WHO Immunisation-Vaccines and Biologicals, US FDA, Health Canada (https://health-products.canada. ca/dpd-bdpp/index-eng.jsp), The Canadian Agency for Drugs and Technologies in Health (CADTH), EMA, Therapeutic Goods Administration, Pharmaceuticals and Medical Devices Agency, ImmunoFacts Vaccines and Immunologic drugs, RxTx (The Canadian Pharmacists Association's e-Therapeutics+and e-Therapeutics+Complete products) and United States Pharmacopoeia and National Formulary, Merck Index, Google Scholar, WHO publications, Global NITAG Network centre, Open Grey and Ministries of Health publications. We may need to contact governmental agencies and committees to gain access to some documents.

The databases that will be searched for studies will be PubMed, MEDLINE (Ovid MEDLINE(R) and Epub Ahead of Print, In-Process \& Other Non-Indexed Citations, Daily and Versions(R)) and EMBASE (Excerpta Medica Database (EMBASE) 1974 to 202026 June (or last version)) to minimise retrieval bias. EMBASE is an international bibliographic science database for biomedical and pharmaceutical product with a comprehensive indexing policy for articles that deal with drugs, and it would be appropriate for this ScR. For RCTs, www.clinicaltrials.gov and the International Clinical trials registry will be searched.

The data sources included in this review are deemed appropriate, given that the evidence will precede and inform the development of the recommendations, which would need to be published, to be considered.

\section{Documents selection and screening}

All monographs are eligible for inclusion and have been uploaded in a file. An Excel sheet gathers vaccine names and weblinks of downloaded monographs. These will be automatically included during third stage of the review where data extraction for off-label vaccines will be performed.
All documents and studies included in public health off-label recommendations-for considered vaccineswill be selected. Moreover, all documents supporting the implementation of the recommendations will be included.

All studies and documents identified in the search will be exported from databases or websites into the EndNote X9 reference manager to eliminate duplication. Unique citations will be exported into DistillerSR for screening. Studies and documents will be reviewed against the selection criteria specified in table 1 for inclusion/exclusion in two stages: the first stage will comprise a review of the title and abstract, where two reviewers, at least one of whom is a content expert and the other a methodology expert, will independently conduct this review to minimise study selection bias; these reviewers will compare and discuss the results for consensus on the exclusion of studies during the first stage of review. Only studies and documents where both reviewers agree as clearly irrelevant to the search will be excluded from the search to maximise the study sensitivity. As the off-label recommendations might not have abstracts, they will be automatically included in the second stage full-text screening.

In the second stage, the same two reviewers will independently review the full text of the included or uncertain studies and other documents to assess the study/ document type, exposure and outcomes. After the first 10 reviews, the two reviewers will meet to calibrate inclusion/exclusion. Disagreements, if any, will be resolved regularlyin batches through discussion during the second stage. A third reviewer will arbitrate if a consensus cannot be reached about a given paper.

After the second stage of the review is completed, bibliographic information of selected articles will be manually searched to find any missing or non-indexed literature. The reviewers will meet to compare results and reach a consensus.

The ScR methodology does not require an evaluation of the quality of studies. However, the quality of evidence is deemed to have been assessed when they were used in the development of recommendations. A report of this assessment is included in the stated objectives of this review and in the identification and summary of evidence.

The study and review processes will be presented in a PRISMA flow chart, ${ }^{31}$ and reasons for exclusion will be provided in the final review report.

\section{Extraction: charting the results}

Data extraction from any type of evidence and research methodology, without restriction to qualitative studies, will be independently undertaken by the two reviewers. A preliminary data extraction of vaccine's indication, concentration of bacteria or virus, route/site, doses and schedule will be performed from all included monographs followed by more extensive data extraction for off-label vaccine used only. Therefore, data will not be extracted and not be included in this ScR if vaccines have not been subject to off-label recommendations. 
Before conducting a complete extraction, a pilot test will be undertaken with a random sample of studies/ documents to assess the quality and the consistency of the data collection by the reviewers and to familiarise themselves with the source of the results. Then, each reviewer will independently extract data by using the same checklist (table 2) and will not be blinded to the authors of the study/document. The reviewers will meet after data extraction for verification purposes: methods, text discrepancy or missing information. This step is paramount in building the final analytical framework considering that data extracted will constitute its mainstays.

A draft charting table was developed to collect the relevant data items from the source and will be refined and continually updated at the review stage.

\section{Synthesis of the results}

The main objective of this review is to synthesise the knowledge on the off-label use of vaccines in a novel initial assessment process ultimately to guide the development of analytical framework for off-label vaccine recommendations. A deductive thematic data analysis will be conducted.

First, the review will commence with a perusal of the vaccine product monographs by presenting information on each vaccine at licensure, which is the study baseline. Then, the review will follow with a case-based analysis for each vaccine by describing the decision process for the initial off-label use of the vaccine and what methods were used; subsequently, off-label vaccine typology and vaccinated typology will be performed on the basis of published recommendations.

The synthesis of data from vaccine off-label recommendations will be either in narrative or tabulated form. For each vaccine, the elements of the decision used to develop the recommendation will be identified: priority questions, research evidence, important factors of evidence appraisal, benefits and harms, costs, feasibility, acceptability, values and preferences of clients or healthcare providers and judgments about criterion or option. A concise summary of pivotal elements that led to the final option will be presented.

In the primary analysis, the study will stratify results by population in accordance with new risk groups with underlying conditions and the healthy population. At the second level, the review will stratify identified papers by study design or type of document, change of schedule, sex, special populations, number of doses, and time of introduction in the vaccination schedule. This analysis will examine the diversity and the possibility of clustering the elements. If any summary or effect measure is assessed and reported in a study, the synthesis will sum up the types of measures that were used and briefly discuss them. When comparing studies, RCTs and observational data will be analysed separately.

Off-label vaccines will be pooled by characteristics: changes in the number of doses in their 'exposure' arm, in the population, in the administration route, or in the indication, followed by pooling by the study design and the type of vaccine. Furthermore, the study will report whether the effect measures documented in studies were from the same calendar time (ie, that the reference group received their vaccinations and were followed during the same calendar time period as the off-label groups).

If the data extracted from the included papers permit diagrammatic presentation, the results will be presented in a dendrogram format that relates to the objectives and question of the review. The results will be clustered by similar evidence, and a narrative description of the data will be presented for the:

- Similarity of study population.

- Similarity of outcome measures.

- Similarity of evidence grade.

- Theoretical concept/no model.

- Similarity of methodology.

- Implementation/no implementation.

\section{ETHICS AND DISSEMINATION}

Ethics approval is not required for this ScR although this manuscript will be part of an entire protocol which will be submitted to the ethics committee. This ScR is the first step for the three phases of this research programme, for a PhD degree. The second phase is a survey where public health experts will answer a questionnaire. Phase 3 includes a focus group in which decision-makers, pharmaceutical industry and the patient/public will be involved. The results will be disseminated through (1) peer-reviewed articles; (2) at conferences. The relevant findings will guide the conceptualisation of (3) an analytical framework for off-label vaccines that will also be submitted to a peer-reviewed journal. Within the global consultation, findings of the review (4) will be presented to stakeholder, policy-maker and public health actors for validation. Iterative consultations are ongoing within the review team.

\section{CONCLUSION}

We present the ScR protocol on the off-label use of vaccines in public programmes, together with an in-depth review of the evidence and concepts from a novel initial analysis of off-label recommendations to identify the findings which are key to decision making in off-label vaccination. To the best of our knowledge, this is the first review to undertake a comprehensive review on the off-label use of vaccines. This study will strengthen the knowledge base of vaccine assessment processes, which are central to the development of novel initial off-label use. Moreover, the mapping of published recommendations will provide an understanding of the extent of off-label vaccine use globally, and on how they facilitate the planning of immunisation programmes. The results of this review will enlighten and support researchers, expert committees, public health actors and policy-makers globally by providing a clear definition of the off-label use of vaccines and guide the conceptualisation of an analytical framework that will 
Table 2 Data extraction sheet

\begin{tabular}{|c|c|c|}
\hline Licensure data & Recommendations & Evidence \\
\hline $\begin{array}{l}\text { Monography } \\
\text { Vaccine preventable disease: }\end{array}$ & $\begin{array}{l}\text { Committee identification } \\
\text { Name of the Advisory committee } \\
\text { Country of the Committee } \\
\text { NITAG member: Yes/No }\end{array}$ & $\begin{array}{l}\text { Qualitative information } \\
\text { Study/document Information: } \\
\text { Authors } \\
\text { Title of publication } \\
\text { Year of Publication } \\
\text { Type of document: } \\
\text { Peer review literature } \\
\text { Unpublished data } \\
\text { Expert opinion } \\
\text { Epidemiological data } \\
\text { Article } \\
\text { Other } \\
\text { Journal name } \\
\text { Study Design } \\
\text { Aims/purpose } \\
\text { Study period } \\
\text { Country(s) in which it took place } \\
\text { Calendar years in follow-up period } \\
\text { Conflicts of Interest declared by authors }\end{array}$ \\
\hline $\begin{array}{l}\text { Identification } \\
\text { Trade name of vaccine } \\
\text { Abbreviation / Proper name } \\
\text { Manufacturer } \\
\text { Licensure date } \\
\text { Date of implementation in a } \\
\text { vaccination programme } \\
\text { country of licensure }\end{array}$ & $\begin{array}{l}\text { Recommendation } \\
\text { Title of the recommendation } \\
\text { Date of publication of the recommendation } \\
\text { Name of journal of publication, or not } \\
\text { Implementation in an immunisation } \\
\text { programme: Yes/No }\end{array}$ & $\begin{array}{l}\text { Population under study: } \\
\text { Initial sample size recruited, } \mathrm{N} \text {, records numbers, } \\
\mathrm{N} \text { and \% Males } \\
\mathrm{N} \text { and \% Females } \\
\text { Age range } \\
\text { Average age } \\
\text { Sample size with full follow-up data available } \\
\mathrm{N} \text { and \% Males } \\
\mathrm{N} \text { and \% Females } \\
\text { Age range } \\
\text { Average age } \\
\text { Medical Comorbidities or Immunosuppressed condition (complete list if different) } \\
\text { HIV/AIDS; } \\
\text { Sickle cell disease, } \\
\text { Nephrotic syndrome, } \\
\text { Asplenia, } \\
\text { Cancer } \\
\text { Asthma } \\
\text { COPD } \\
\text { Diabetes } \\
\text { Thyroid disorders } \\
\text { IBD } \\
\text { Lifestyle factors: } \\
\text { Exposure to tobacco smoke. } \\
\text { Overweight } \\
\text { Malnutrition } \\
\text { Day care attendance } \\
\text { Lack of breastfeeding }\end{array}$ \\
\hline $\begin{array}{l}\text { Typology } \\
\text { Therapeutic indication } \\
\text { Posology, doses, no of shots in } \\
\text { routine series } \\
\text { Approved ages } \\
\text { Specific population groups, sex } \\
\text { Method of administration }\end{array}$ & $\begin{array}{l}\text { Discussion structure } \\
\text { Use of a framework Yes/No } \\
\text { Name of the framework } \\
\text { Use of Theoretical concept Yes/No } \\
\text { Name of the concept } \\
\text { Use of a standard operation procedure Yes/No } \\
\text { Name of the SOP }\end{array}$ & $\begin{array}{l}\text { Off-label vaccine intervention (Exposure): } \\
\text { Name of vaccine } \\
\text { Quantity of type of strains protected against } \\
\text { Dose per shot } \\
\text { No and timing of doses } \\
\text { Measurement instrument/method, specific } \\
\text { Calendar years intervention measured } \\
\text { Immunisation schedule } \\
\text { Group of the population } \\
\text { Off-label characteristics } \\
\text { Outcome measure: } \\
\text { Immunogenicity serological threshold antibody levels } \\
\text { Vaccine effectiveness (endpoint measure) } \\
\text { Vaccine impact } \\
\text { Vaccine safety } \\
\text { Immunologic non-inferiority (indicate } \delta \text { ) } \\
\text { Incidence of the disease } \\
\text { Clinical criteria used for the disease } \\
\text { Method of disease measurement/diagnosis } \\
\text { Methods: } \\
\text { Population description (inclusion/exclusion) } \\
\text { Randomisation process for RCTs (RCTs) } \\
\text { Assessment of exposure status (cohort) } \\
\text { Age groups: N, \% in each } \\
<1 ; 1-4 ; 5-9 ; 10-14 ; 15-18 ; \\
19-24 ; 25-29 ; 30-39 ; 40-59 ; \\
\text { Sex; } \\
\text { Immuno } \% \text { F - N, \% M) } \\
\text { Prior vaccination } \\
\text { Vaccination interval different for intervention versus control arm }\end{array}$ \\
\hline
\end{tabular}


Table 2 Continued

\begin{tabular}{|c|c|c|}
\hline Licensure data & Recommendations & Evidence \\
\hline $\begin{array}{l}\text { Composition } \\
\text { Antigen } \\
\text { Adjuvant } \\
\text { Protein } \\
\text { Other components } \\
\text { Live or attenuated vaccine } \\
\text { Bacteria, virus, toxoid, } \\
\text { protozoan } \\
\text { Wild strain or not, no of strains }\end{array}$ & 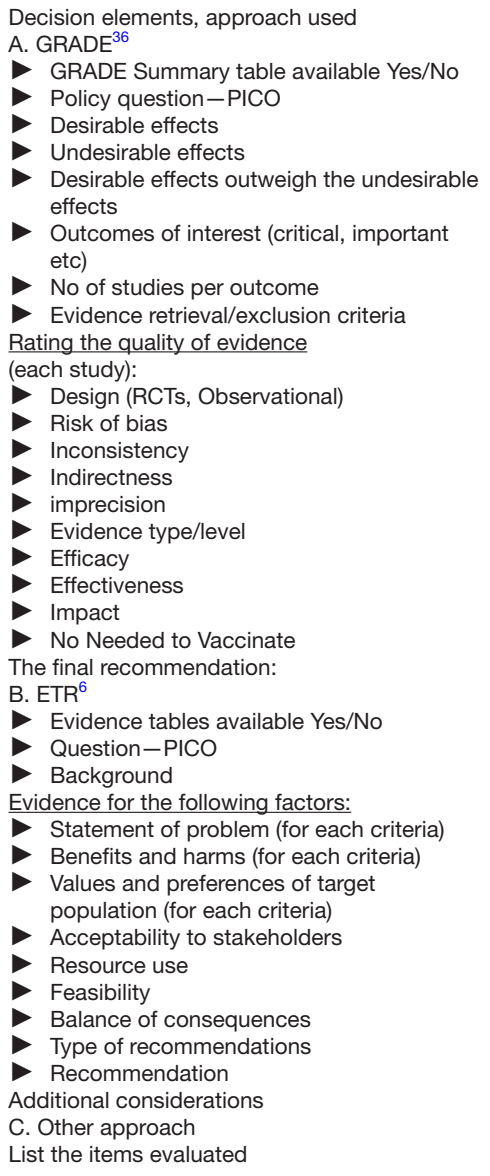 & $\begin{array}{l}\text { Quantitative information (for studies) } \\
\text { Effect measures (yes/no) } \\
\text { OR, RR or HR rates } \mathrm{n} / \mathrm{N} \\
\mathrm{SD} \\
\mathrm{Cl} \\
\text { Variance } \\
\text { Adjusted/unadjusted }\end{array}$ \\
\hline \multicolumn{3}{|l|}{$\begin{array}{l}\text { Contraindication } \\
\text { Population } \\
\text { Sex } \\
\text { Age group } \\
\text { Fertility, pregnancy and lactation }\end{array}$} \\
\hline \multicolumn{3}{|l|}{$\begin{array}{l}\text { Immunogenicity } \\
\text { Serological threshold } \\
\text { Antibody level }\end{array}$} \\
\hline \multicolumn{3}{|c|}{$\begin{array}{l}\text { Other information } \rightarrow \text { accessible written evidence } \\
\text { Vaccine updates } \\
\text { Others }\end{array}$} \\
\hline
\end{tabular}

COPD, Chronic obstructive pulmonary disease; EtR, evidence to recommendations; GRADE, Grading of Recommendations Assessment, Development and Evaluation; IBD, Inflammatory bowel disease; NITAG, National Immunisation Technical Advisory Group; PICO, patient/Population, Intervention, Comparison and Outcomes; RCTs, randomised controlled trials; SOP, Standard operating procedure.

be used for the assessment of evidence in the development of future recommendations for the off-label use of vaccines in public programmes. Furthermore, we anticipate that the findings of this $\mathrm{ScR}$ will inspire research into the off-label use of agents beyond vaccination, where off-label indications play a considerable role.

Acknowledgements We would like to thank Monique Clar for her assistance in the development of the search strategy.

Contributors DD ( $\mathrm{PhD}$ candidate) participated in conceptualisation of the project, researched and developed all aspects of the project methodology, design and manuscript and approved the final version as submitted. CQ (research director) participated in conceptualisation of the project, critically reviewed and commented on the whole manuscript and approved the final version of the protocol.

Funding The authors have not declared a specific grant for this research from any funding agency in the public, commercial or not-for-profit sectors.
Competing interests DD is a scientific advisor to the Directorate of Biological Risks and Occupational Health, Institut National de Santé Publique du Québec, Québec, Canada. CQ is the chair and a previous committee member of the National Advisory Committee on Immunisation (NACl)-Canadian NITAG-Centre for Immunisation and Respiratory Infectious Diseases, Public Health Agency of Canada, Ottawa, Canada.

Patient consent for publication Not required.

Provenance and peer review Not commissioned; externally peer reviewed.

Supplemental material This content has been supplied by the author(s). It has not been vetted by BMJ Publishing Group Limited (BMJ) and may not have been peer-reviewed. Any opinions or recommendations discussed are solely those of the author(s) and are not endorsed by BMJ. BMJ disclaims all liability and responsibility arising from any reliance placed on the content. Where the content includes any translated material, BMJ does not warrant the accuracy and reliability of the translations (including but not limited to local regulations, clinical guidelines, terminology, drug names and drug dosages), and is not responsible for any error and/or omissions arising from translation and adaptation or otherwise. 
Open access This is an open access article distributed in accordance with the Creative Commons Attribution Non Commercial (CC BY-NC 4.0) license, which permits others to distribute, remix, adapt, build upon this work non-commercially, and license their derivative works on different terms, provided the original work is properly cited, appropriate credit is given, any changes made indicated, and the use is non-commercial. See: http://creativecommons.org/licenses/by-nc/4.0/.

\section{ORCID iDs}

Dieynaba Diallo http://orcid.org/0000-0002-3650-5815

Caroline Quach http://orcid.org/0000-0002-1170-9475

\section{REFERENCES}

1 World Health Organization. The world health report 1996 - Fighting disease, fostering development. Geneva: WHO Library Cataloguing in Publication Data, 1996.

2 World Health Organization. Disease burden and mortality estimates. cause-specific mortality, 2000-2016. global health estimates 2016: disease burden by cause, age, sex, by country and by region, 20002016. Geneva: World Health Organization, 2018.

3 Statistics Canada. Table 13-10-0141-01 deaths, by cause, chapter I: certain infectious and parasitic diseases (A00 to B99), 2020.

4 World Health Organization - UNICEF. Global and regional immunization profile 2018. In: 2019 global summary ed. Geneva: World Health Organization, ed, 2019.

5 Evaluation Directorate-Health Canada and the Public Health Agency of Canada. Evaluation of the biologics program 1999-2000 to 20122013 Canada. 174, 2014.

6 U.S. Food and Drug Administration. Food and drugs - Requirements on content and format of labeling for human prescription drug and biological products. Department of health and human services, ed, 2006.

7 Meissner HC, Farizo K, Pratt D, et al. Understanding FDA-approved labeling and CDC recommendations for use of vaccines. Pediatrics 2018;142:e20180780.

8 Neels P, Southern J, Abramson J, et al. Off-label use of vaccines. Vaccine 2017;35:2329-37.

9 National Advisory Committee on Immunization (NACl). Update on the recommendations for the routine use of pneumococcal conjugate vaccine for infants. An Advisory Committee statement (ACS). Can Commun Dis Rep 2006;32:1-6.

10 Pasteur S. REPEVAX - Summary of Product Characteristics (SmPC) Electronic Medicines Compendium (emc). UK, 2012.

11 Pasteur S. REPEVAX, suspension for injection, in pre-filled syringe. The electronic medicines compendium (emc), 2019.

12 US Food and Drug Administration. February 3, 2006 Approval Letter - RotaTeq. U.S.A: Department of health and human services, 2006.

13 Merck \& Co. Inc.. RotaTeq (Rotavirus Vaccine, Live, Oral, Pentavalent) - Highlights of prescribing information - Full prescribing information., 2006. Available: https://www.merck.com/product/usa/ pi_circulars/r/rotateq/rotateq_pi.pdf [Accessed 18 Jan 2020].

14 Parashar UD, Alexander JP, Glass RI, et al. Prevention of rotavirus gastroenteritis among infants and children. Recommendations of the Advisory Committee on immunization practices (ACIP). MMWR Recomm Rep 2006;55:1-13.

15 European Medicines Agency. Rotarix - rotavirus vaccine, live, attenuated - Summary of the European public assessment report (EPAR). Belgium: European Union, 2006.

16 Glaxosmithkline Inc. Summary of product characteristics. ROTARIXHuman rotavirus, live, attenuated, oral vaccine, 2006. Available: https://ca.gsk.com/media/1216129/rotarix.pdf [Accessed 18 Jan 2020].
17 US Food and drug administration. Summary Basis for Regulatory Action - RotaTeq $囚 /$ Rotavirus Vaccine, Live, Oral, Pentavalent. U.S.A, 2017.

18 Dai X, Bai R, Jian M, et al. Immunogenicity of different dosing schedules of the human live attenuate rotavirus vaccine (RV1) in infants and children: a meta-analysis. Hum Vaccin Immunother 2019:15:1228-36.

19 Alicino C, Paganino C, Orsi A, et al. The impact of 10-valent and 13-valent pneumococcal conjugate vaccines on hospitalization for pneumonia in children: a systematic review and meta-analysis. Vaccine 2017;35:5776-85.

20 Yakely AE, Avni-Singer L, Oliveira CR, et al. Human papillomavirus vaccination and anogenital warts: a systematic review of impact and effectiveness in the United States. Sex Transm Dis 2019;46:213-20.

21 Karafillakis E, Hassounah S, Atchison C. Effectiveness and impact of rotavirus vaccines in Europe, 2006-2014. Vaccine 2015;33:2097-107.

22 Kraicer-Melamed H, O'Donnell S, Quach C. The effectiveness of pneumococcal polysaccharide vaccine 23 (PPV23) in the general population of 50 years of age and older: a systematic review and meta-analysis. Vaccine 2016;34:1540-50.

23 Markowitz LE, Drolet M, Perez N, et al. Human papillomavirus vaccine effectiveness by number of doses: systematic review of data from national immunization programs. Vaccine 2018;36:4806-15.

24 Higgins JPT, Soares-Weiser K, López-López JA, et al. Association of BCG, DTP, and measles containing vaccines with childhood mortality: systematic review. BMJ 2016:355:i5170.

25 Theodoratou E, Johnson S, Jhass A, et al. The effect of Haemophilus influenzae type $b$ and pneumococcal conjugate vaccines on childhood pneumonia incidence, severe morbidity and mortality. Int J Epidemiol 2010;39(Suppl 1):i172-85.

26 Top KA, Esteghamati A, Kervin M, et al. Governing off-label vaccine use: an environmental scan of the global national immunization technical Advisory group network. Vaccine 2020;38:1089-95.

27 Gallagher K, Cocks N, Mounier-Jack S. Scoping views on a single dose human papillomavirus (HPV) vaccine schedule amongst policy makers in low and middle-income countries 2018.

28 Chocarro L, Duclos P, Senouci K, et al. Consultation on interactions between national regulatory authorities and national immunization technical Advisory groups. Expert Rev Vaccines 2011;10:1265-70.

29 MacDonald NE, Duclos P, Wichmann O, et al. Moving forward on strengthening and sustaining national immunization technical Advisory groups (NITAGs) globally: recommendations from the 2nd global NITAG network meeting. Vaccine 2017;35:6925-30.

30 Peters MDJ GC, Mclnerney P, Baldini Soares C, et al. Chapter 11: Scoping Reviews. In: Aromataris E, Munn Z, eds. Joanna Briggs Institute Reviewer's Manual. The Joanna Briggs Institute, 2017. https://reviewersmanual.joannabriggs.org/

31 Tricco AC, Lillie E, Zarin W, et al. PRISMA extension for scoping reviews (PRISMA-ScR): checklist and explanation. Ann Intern Med 2018;169:467-73.

32 Diallo D, Quach C. Evaluation of the decision-making process underlying the novel initial off-label use of vaccines: a scoping review protocol. Open science framework (registries) 2020.

33 Canadian Agency for Drugs Technologies in Health (CADTH). Grey Matters: a practical tool for searching health-related grey literature Internet. 2018. Ottawa, 2019.

34 Mesgarpour B, Müller M, Herkner H. Search strategies to identify reports on "off-label" drug use in EMBASE. BMC Med Res Methodol 2012;12:190.

35 Mesgarpour B, Müller M, Herkner H. Search strategies-identified reports on "off-label" drug use in MEDLINE. J Clin Epidemiol 2012;65:827-34.

36 Lee G, Carr W, ACIP Evidence-Based Recommendations Work Group. Updated framework for development of evidence-based recommendations by the Advisory Committee on immunization practices. MMWR Morb Mortal Wkly Rep 2018;67:1271-2. 